\title{
Music and emotion: Electrophysiological correlates of the processing of pleasant and unpleasant music
}

\author{
DANIELA SAMMLER, MAREN GRIGUTSCH, THOMAS FRITZ, AND STEFAN KOELSCH
}

Max Planck Institute for Human Cognitive and Brain Sciences, Leipzig, Germany

\begin{abstract}
Human emotion and its electrophysiological correlates are still poorly understood. The present study examined whether the valence of perceived emotions would differentially influence EEG power spectra and heart rate (HR). Pleasant and unpleasant emotions were induced by consonant and dissonant music. Unpleasant (compared to pleasant) music evoked a significant decrease of $\mathrm{HR}$, replicating the pattern of $\mathrm{HR}$ responses previously described for the processing of emotional pictures, sounds, and films. In the EEG, pleasant (contrasted to unpleasant) music was associated with an increase of frontal midline (Fm) theta power. This effect is taken to reflect emotional processing in close interaction with attentional functions. These findings show that Fm theta is modulated by emotion more strongly than previously believed.
\end{abstract}

Descriptors: Emotion, Music, Dissonance, EEG, Fm theta, Heart rate

During the past 15 years, numerous imaging studies have provided considerable insights into the neural basis of pleasant and unpleasant emotions (e.g., Dalgleish, 2004; Davidson \& Irwin, 1999; Phan, Wager, Taylor, \& Liberzon, 2002). On the other hand, electrophysiological correlates of pleasant emotions remain largely unknown, even though EEG provides an ecologically more valid recording situation (sitting upright in a silent room) than fMRI (loud scanner noise and narrow space) or PET (application of radioactive substances). Furthermore, due to the high temporal resolution of EEG, it is possible to study neuronal processes at different time scales, that is, frequency bands that are related to different mental functions and most presumably also to emotional states. The present study aimed to elucidate whether and in which frequency bands EEG would raise reliable correlates of emotion processing.

Traditionally, the human EEG power spectrum is divided into at least five frequency bands: delta, theta, alpha, beta, and gamma (Niedermeyer, 1999). In the past decades, each frequency band has been related to specific functions, which will be briefly

We thank Sven Gutekunst for his technical support during the preparation of this experiment, Sylvia Stasch for her great help during data acquisition, Kerstin Flake for her assistance with the figures, and Nikolaus Steinbeis and Katrin Schulze for fruitful discussion. Furthermore, we thank the editor and two anonymous reviewers for their valuable comments on a former version of this article.

Examples of the stimuli are available at http://www.stefan-koelsch.de/ Music_Emotion1.

Address reprint requests to: Daniela Sammler, Max Planck Institute for Human Cognitive and Brain Sciences, Junior Research Group "Neurocognition of Music", Stephanstrasse 1a, D-04103 Leipzig, Germany. E-mail: sammler@cbs.mpg.de. reviewed for the theta, alpha, and beta frequency range in the following sections.

\section{Theta (4-8 Hz)}

The adult human theta rhythm has (at least) two manifestations (Schacter, 1977): The first type is characterized by a widespread scalp distribution and has been mainly observed during drowsiness (e.g., due to sleep deprivation) and states of low-level alertness resulting in inefficient information processing. The second type is often referred to as "frontal midline theta" (Fm theta) because of its typical scalp topography. Fm theta has been observed during a large variety of tasks (for overviews, see Inanaga, 1998; Schacter, 1977) such as mental calculation (e.g., Asada, Fukuda, Tsunoda, Yamaguchi, \& Tonoike, 1999; Sasaki, Tsujimoto, Nambu, Matsuzaki, \& Kyuhou, 1994), working memory and learning (e.g., Gabriel, Sparenborg, \& Stolar, 1986; Gevins, Smith, McEvoy, \& Yu, 1997; Jensen \& Tesche, 2002; Klimesch, Schack, \& Sauseng, 2005; McEvoy, Pellouchoud, Smith, \& Gevins, 2001; Onton, Delorme, \& Makeig, 2005), error processing (Luu \& Tucker, 2001; Luu, Tucker, Derryberry, Reed, \& Poulsen, 2003; Luu, Tucker, \& Makeig, 2004), and meditation (e.g., Aftanas \& Golocheikine, 2001; Kubota et al., 2001). Therefore, Fm theta has been most often interpreted as a correlate of heightened mental effort and sustained attention required during a multitude of operations.

Studies applying EEG/MEG source modeling on different (nonemotional) mental tasks provide converging evidence that dipoles within medial frontal cortex (MFC), especially within dorsal anterior cingulate cortex (ACC; BA 24/32), account for frontal midline theta activity recorded at the scalp (Asada et al., 1999; Gevins et al., 1997). Recently, Fm theta activity during rest 
has been linked with the cerebral metabolism in the rostral ACC (BA 24/32) by means of combined EEG and PET measurements (Pizzagalli, Oakes, \& Davidson, 2003). Furthermore, intracranial recordings in humans revealed rhythmic theta activity within the ACC (Nishida et al., 2004; Uchida, Maehara, Hirai, Kawai, $\&$ Shimizu, 2003). Apart from ACC, theta oscillations were also reported within septo-hippocampal and other subcortical limbic structures as well as entorhinal cortex (Arnolds, Lopes da Silva, Aitink, Kamp, \& Boeijinga, 1980; Bland \& Oddie, 1998; Lopes da Silva, 1992; Meador et al., 1991; Vinogradova, 1995). However, due to their closed field characteristics (Klee \& Rall, 1977) the contribution of these structures to Fm theta is most presumably rather minor.

The ACC is a part of the limbic system (Mega, Cummings, Salloway, \& Malloy, 1997) and receives strong input from the amygdala, hippocampus, and parahippocampal gyrus (Devinsky, Morrell, \& Vogt, 1995; Paus, 2001). The ACC has, thus, been largely implicated in emotional processing, apart from other functions such as attention, autonomic control, and motor control (Bush, Luu, \& Posner, 2000; Critchley et al., 2003; Devinsky et al., 1995). In support of this, several studies investigating emotions with music consistently reported activations of anterior cingulate structures during the processing of pleasant emotions (Blood \& Zatorre, 2001; Blood, Zatorre, Bermudez, \& Evans, 1999).

These findings that Fm theta originates from the ACC, that the ACC is part of a neural "emotion circuit," and that the ACC is activated by pleasant music raise the highly pertinent question of whether Fm theta may be related to emotional processing. So far, only a very few studies have addressed this issue. Recently, Aftanas and Golocheikine (2001) reported an increase of Fm theta power during "blissful positive states" achieved during meditation. The Fm theta power was positively correlated not only with scores of internalized attention but also with subjective scores of the pleasantness of the emotional experience. Furthermore, two studies on the relationship between Fm theta and anxiety consistently reported negative correlations between Fm theta during mental tasks and anxiety measures (e.g., scores of the state-trait anxiety inventory; Mizuki et al., 1992; Suetsugi et al., 2000). Based on these findings, we hypothesized that listening to consonant (i.e., pleasant) music would elicit an increase of Fm theta power.

\section{Alpha (8-12 Hz)}

Reports on EEG alpha activity distinguish at least three different types of alpha rhythms differing in topography and function (see, e.g., Hari \& Salmelin, 1997; Hari, Salmelin, Makela, Salenius, \& Helle, 1997; Klimesch, 1999). First, the classical posterior alpha rhythm originating from parieto-occipital cortex is strongly dependent on attentional factors and the vigilance of the subject. Second, the Rolandic mu rhythm that is especially dominant at central electrodes and most presumably originates from somatosensory cortex is commonly related to movement and movement preparation (Crone et al., 1998; Hari et al., 1997; Pfurtscheller \& Lopes da Silva, 1999). Third, the tau rhythm is generated within auditory cortices and modulated by auditory stimulation (Hari \& Salmelin, 1997; Salmelin \& Hari, 1994; Tiihonen et al., 1991). Note that, in general, alpha power and brain activity are inversely related (i.e., a decrease in alpha power indicates an increase in brain activity). Apart from these functions, the alpha rhythms are broadly linked with perceptual processing and memory tasks (e.g., Başar, 1999; Klimesch, 1999;
Klimesch et al., 2005; Ward, 2003), and have been largely implicated in emotional processing (Aftanas, Koshkarov, Pokrovskaja, Lotova, \& Mordvintsev, 1996; Davidson, 1995; Schmidt \& Trainor, 2001; Tsang, Trainor, Santesso, Tasker, \& Schmidt, 2001).

A considerable body of research focused on the relation between emotional processing and frontal alpha asymmetry leading to the development of the "hemispheric valence hypothesis" (Davidson, 1995; Heilman, 1997). This hypothesis states that positive approach-related emotions are mainly processed in left frontal brain areas, whereas negative withdrawal-related emotions rather engage right frontal brain regions. In the EEG this is reflected by an asymmetric decrease of alpha power according to the perceived emotion, that is, a decrease of left frontal alpha power during positive emotions and a decrease of right frontal alpha power during negative emotions. Corresponding studies either investigate the traitlike frontal alpha asymmetry in the resting EEG of healthy subjects and different patient populations or the asymmetry of anterior cortical activity during stimulusinduced emotional states (for reviews, see Coan \& Allen, 2004; Davidson, 1995).

A number of studies used different types of stimuli to induce emotions, for example, positive or negative film clips (Davidson, Ekman, Saron, Senulis, \& Friesen, 1990), reward or punishment (Sobotka, Davidson, \& Senulis, 1992), and picture viewing (Aftanas, Varlamov, Pavlov, Makhnev, \& Reva, 2001). These studies corroborated the relation between emotional state and forebrain EEG asymmetry (but see also Hagemann, 2004; Hagemann, Naumann, Becker, Maier, \& Bartussek, 1998; Heller \& Nitschke, 1998; Reid, Duke, \& Allen, 1998). Recently, also two EEG studies on musically induced emotions provided evidence in favor of the frontal alpha asymmetry: Schmidt and Trainor (2001) and Tsang et al. (2001) presented happy and sad musical excerpts and found decreased alpha power at left frontal electrodes during happy music, whereas sad music was associated with a more pronounced alpha power decrease at right frontal leads. Similarly, Altenmüller, Schürmann, Lim, and Parlitz (2002), who did not focus on alpha power but evaluated DC potentials during pleasant and unpleasant music and sounds, reported a frontal brain asymmetry. Based on these findings, we expected a stronger left frontal alpha power decrease during consonant pieces and a stronger right frontal alpha power decrease during dissonant excerpts.

\section{Beta $(13-30 \mathrm{~Hz})$}

Widely distributed EEG beta activity is thought to be related to increased alertness and cognitive processes (Steriade, 1999). It has been shown that beta power decreases during non-REM sleep proportionally with growing delta power (the latter indicating increasing sleep depth). Only a few studies investigated the association between beta power and emotional processing (e.g., Aftanas, Reva, Savotina, \& Makhnev, 2006; Sebastiani, Simoni, Gemignani, Ghelarducci, \& Santarcangelo, 2003). They mainly reported an increase in beta power following an unspecific increase of emotional arousal (mainly independent of valence). Apart from this, a circumscribed centrally distributed cortical beta rhythm is strongly related to motor functions and has been found to originate from primary motor cortex (Crone et al., 1998; Hari et al., 1997; Pfurtscheller \& Lopes da Silva, 1999), as well as from the supplementary motor area (Pfurtscheller, Woertz, Supp, \& Lopes da Silva, 2003).

In the present study, we employed an experimental paradigm that had already been used in a previous fMRI study (Koelsch, 
Fritz, von Cramon, Muller, \& Friederici, 2006). Pleasant emotions were induced by joyful, mainly consonant musical tunes. Electronically manipulated (permanently dissonant) counterparts of these pieces were used to induce unpleasant emotions. This approach was based on the well-documented finding that sensory dissonance (in the sense of the roughness of a stimulus; see Bigand, Parncutt, \& Lerdahl, 1996) is generally perceived as unpleasant (Blood et al., 1999; Koelsch et al., 2006) even by 4- and 6-month-old babies (Trainor \& Heinmiller, 1998; Zentner $\&$ Kagan, 1998). The present paradigm, thus, allows the investigation of emotional processing with music independent of personal preferences of musical styles.

In the mentioned fMRI study (Koelsch et al., 2006), dissonant (compared to consonant) musical stimuli elicited activity changes in limbic and paralimbic brain structures (such as amygdala, hippocampus, parahippocampal gyrus, and temporal pole) known to be involved in emotional processing. Additional activations in response to the consonant (compared to dissonant) music were observed in the larynx representation within the Rolandic operculum (BA43) and inferior frontal gyrus (BA44i, BA45/46). In almost all of the mentioned structures, activations increased in the course of each musical stimulus, indicating that the effects of emotional processing dynamically evolve over time (see Koelsch et al., 2006). Similarly, a study from Krumhansl (1997), which investigated the time course of autonomic responses during the listening to music of different affective styles, found that the strongest physiological effects elicited by the emotional music pieces tended to increase over time. To date, there is no EEG study investigating the temporal dynamics of emotional processing. We hypothesized that also in the EEG effects would be stronger during the second than during the first half of each musical piece.

In addition to the EEG, the present study included the measurement of heart rate $(\mathrm{HR})$ to investigate autonomic responses related to the experience of emotions. Krumhansl (1997) reported significantly different physiological patterns during listening to musical pieces chosen to represent sadness, happiness, and fear, for example, a decrease of HR during sad musical excerpts. Bradley and Lang induced emotions with pictures from the International Affective Picture System (IAPS; Lang, Bradley, \& Cuthbert, 1999). One major finding was a more pronounced, parasympathetically driven overall deceleration of HR during unpleasant than during pleasant emotions in healthy participants (but see also Cook \& Turpin, 1997; Lang, Bradley, \& Cuthbert, 1997, for a description of different HR effects in phobic subjects). In other studies, this HR deceleration following unpleasant stimuli was basically replicated for highly arousing environmental sounds from the International Affective Digitized Sounds database (IADS; Bradley \& Lang, 1999, 2000a) as well as for short film clips presented with or without music (Ellis \& Simons, 2005). Based on the above mentioned findings, we hypothesized that the deceleration of HR would be more pronounced during listening to dissonant (compared to consonant) musical pieces.

In summary, the present study was designed to investigate brain mechanisms underlying the processing of (un)pleasant musically induced emotions. We compared (a) subjective ratings on the emotional valence of consonant/dissonant musical pieces, (b) HR changes, and (c) EEG power in different frequency bands to identify neurophysiological correlates of emotional processing and to investigate their temporal dynamics. It was hypothesized that listening to consonant (i.e., pleasant) music would elicit an increase of Fm theta power. During consonant pieces, a greater left frontal alpha power decrease was predicted, whereas dissonant excerpts were hypothesized to evoke a stronger right frontal decrease of alpha power. The beta band was explored without directed predictions. Effects within EEG were thought to increase in the time course of the musical stimuli. HR was expected to significantly decrease during dissonant pieces.

\section{Methods}

\section{Participants}

Data were obtained from 18 students (aged 20-30, mean age 24.67 years, 8 men). Participants were right-handed (mean laterality quotient $=99.44 \%$ ) according to the Edinburgh Handedness Inventory (Oldfield, 1971). Participants were nonmusicians who had no formal musical training (besides typical school education) and had never learned to play a musical instrument. All participants reported normal hearing and no history of neurological disease.

\section{Stimuli}

Participants were presented with two categories of musical pieces differing in their degree of dissonance. Stimuli were the same as the ones used in a previous fMRI study (Koelsch et al., 2006): Consonant (i.e., pleasant) pieces comprised 10 excerpts of joyful instrumental dance tunes of the past four centuries available on commercial CDs. ${ }^{1}$ On average, stimuli had a duration of approximately $1 \mathrm{~min}$ (range: $44.87-67.99 \mathrm{~s}, M=58.15 \mathrm{~s}$ ) and were presented with a mean tempo of 120 beats per minute (bpm; range: $64.1-154.13 \mathrm{bpm}, M=120.19 \mathrm{bpm}$ ). To rule out that differences of behavioral or electrophysiological measures between the first and second half of the excerpts are due to tempo variations, it was controlled that the single excerpts did not speed up or slow down over time. A $t$ test for paired samples on the average tempo of the first (i.e., $0-22 \mathrm{~s} ; M=119.78 \mathrm{bpm}$ ) and second half (i.e., 22-44 s; $M=120.51 \mathrm{bpm}$ ) of the stimuli revealed a constant tempo in the time course of the pieces, $t(9)=0.46 ; p>.6$.

Dissonant (i.e., unpleasant) stimuli were electronically manipulated counterparts of the consonant excerpts: Using Cool Edit Pro (Syntrillium) software, a new sound file was created in which the original consonant version of each piece was played simultaneously with two pitch-shifted versions of the same excerpt, the pitch-shifted versions being a tritone below and one tone above the original pitch (sound samples of the stimuli are available at http://www.stefan-koelsch.de/Music_Emotion1). Importantly, consonant and dissonant versions of each piece were, thus, identical in their dynamic outline, their rhythmic structure and tempo, and their melodic contour. Consequently, it is not possible that any of these stimulus properties contribute to differences in autonomic and neural effects between consonant and dissonant pieces.

To evaluate the impact of the stimulus material on individually perceived emotions and the stability of this effect in the time course of the musical pieces, a behavioral experiment was

${ }^{1}$ A. Dvořák: Slavonic Dance No. 8 in G Minor (Op. 46); A. Dvořák: Slavonic Dance No. 9 in B Major (Op. 72); J. S. Bach: Badinerie (Ouverture No. 2, BMV 1067); J. S. Bach: Bourrée (Ouverture No. 1, BWV 1066); J. S. Bach: Rejouissance (BWV 1069); F. Canaro: La Punalada (CD-ASIN: B00000DXZQ); J. Pastorius: Soul intro ("The Chicken", CD-ASIN: B0000C24JN); P. F. Caroubel: Volte (CD-ASIN: B0000247QD); Anonymus: Entrée Courante (CD-ASIN: B0000247QD); V. Haußmann: Catkantei (CD-ASIN: B0000247QD). 


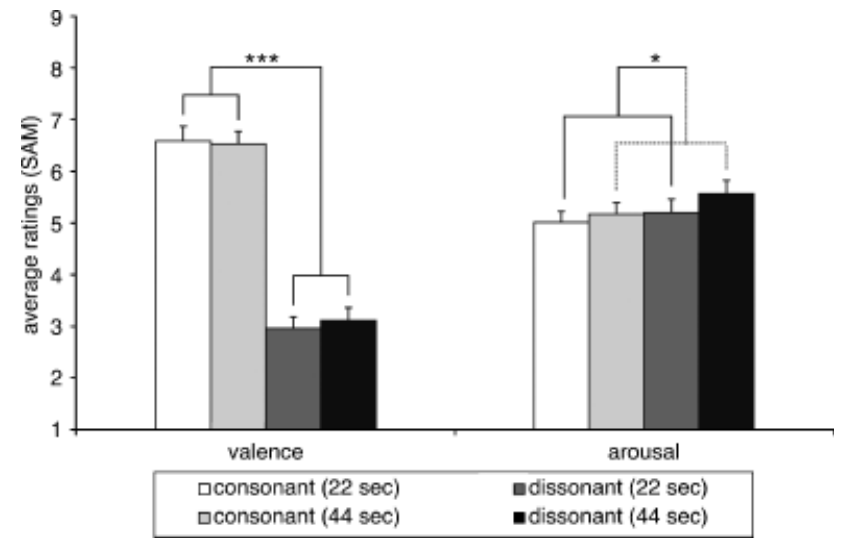

Figure 1. Average ratings of valence and arousal for short (22s) and long (44 s) consonant and dissonant musical excerpts (error bars indicate $S E M)$. Valence ratings differed significantly between consonant and dissonant pieces, independent of stimulus duration (left). In contrast, both stimulus categories were comparable in arousal (right). However, arousal increased with stimulus duration (right; ${ }^{*} p<.05 ; * * * p<.001$ ).

conducted. Twenty-two nonmusicians (aged 20-29, mean age 24.64 years, $11 \mathrm{men}$ ) were randomly presented with the initial 22 $\mathrm{s}$ and $44 \mathrm{~s}$ (corresponding to the maximum common duration) of all musical pieces. After each excerpt, participants rated how (un)pleasant and aroused they felt using the Self-Assessment Manikin (SAM; Bradley \& Lang, 1994). Two-way repeated measures ANOVAs with factors Condition (consonant vs. dissonant) and Duration (22 s vs. 44 s) were calculated separately for valence and arousal ratings (see Figure 1).

Consonant pieces were rated as significantly more pleasant than their dissonant versions (main effect of Condition: $F[1,21]=98.29, p<.001$ ), supporting the relation between sensory dissonance and unpleasantness. Most importantly, this effect occurred independently of stimulus duration (no main effect of Duration: $p>.4$; no interaction: $p>.1$ ). The analysis of arousal ratings revealed that consonant and dissonant pieces did not significantly differ in arousal (no main effect of Condition: $p>.1$ ), but arousal increased similarly in both the consonant and the dissonant conditions with increasing stimulus duration (main effect of Duration: $F[1,21]=5.40, p<.04$; no interaction: $p>.6$ ). Accordingly, differences in psychophysiological effects between consonant and dissonant pieces cannot be attributed to a different arousal level of the stimulus classes. Increasing effect strengths during the second compared to the first half of musical excerpts that occur for both consonant and dissonant music may be related to a higher arousal level.

\section{Procedure}

Participants were presented with all musical stimuli 2-5 days prior to the EEG experiment as well as during the course of EEG preparation to ensure that all participants were equally familiar with the stimulus material. According to the statements of the participants after the first exposure to the musical excerpts, none of the pieces sounded familiar to them. During the experiment, each musical excerpt was presented once; consonant and dissonant stimuli were presented in alternating order, with consonant and dissonant versions of the same piece never following each other. Furthermore, the experiment comprised six baseline periods without stimulation. An auditory cue (consisting either of four short ascending sine wave tones in music trials or of a low

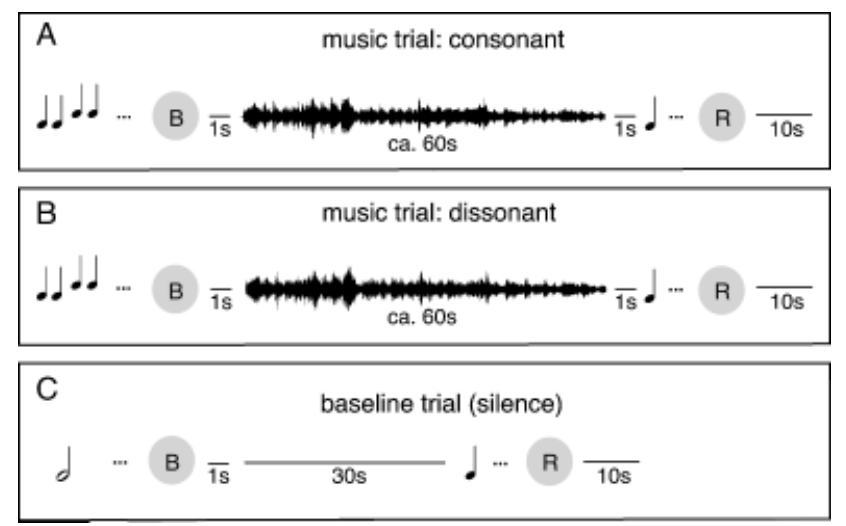

Figure 2. Time course of (A) consonant music, (B) dissonant music, and (C) baseline trials. After an initial auditory cue, the participants started the trial with a button press (B). Musical excerpts and the silent period were followed by a short signal tone that prompted participants to rate (R) their current emotional state. A 10-s pause was added before the presentation of the next auditory cue.

single sine wave tone in baseline trials) prompted participants to start the trial by pressing a button. In music trials, listeners were presented with a musical excerpt. Baseline trials consisted of a 30 -s silent period. At the end of each trial (i.e., after each musical excerpt and silent period), a signal tone prompted participants to rate how (un)pleasant they felt during the music/silence by pressing a button according to a 6-point scale (with 1 corresponding to very unpleasant and 6 corresponding to very pleasant). That is, participants were asked to judge their individually experienced emotion, not to evaluate the emotion expressed by the music (see also Koelsch et al., 2006). Each rating was followed by a 10-s pause until the next auditory cue was presented (Figure 2). Behavioral ratings were averaged for the consonant and dissonant excerpts separately for each participant. Subsequently, ratings from all subjects were compared at the group level with $t$ tests for paired samples.

During the EEG measurement, participants sat in a comfortable chair in an electrically shielded room. Musical pieces were presented via loudspeakers at a comfortable volume using Presentation 0.52 software. Participants were asked to listen carefully to the music and to keep their eyes closed during the whole experiment, making the measurements more comfortable than with a fixation cross. To be consistent with the experiment of Koelsch et al. (2006) and to control whether listeners paid attention not only to the consonant, but also to the dissonant stimuli, participants were asked to tap the beat of the music (i.e., the meter) with their right index finger. To make sure that participants understood the instructions, they were given a minimum of 10 practice trials.

\section{EEG Recordings and Data Analysis}

The electroencephalogram (EEG) was recorded with $\mathrm{Ag} / \mathrm{AgCl}$ electrodes from 63 electrode sites placed according to the extended international 10-20 system (Electro Cap International Inc., Eaton, USA). Left mastoid (M1) served as reference; the ground electrode was located on the sternum. To control for artifacts caused by eye movements, horizontal and vertical electrooculograms (EOG) were recorded bipolarly with electrodes placed on the outer canthus of each eye (horizontal EOG), the tip of the nose, and FPZ (vertical EOG). Signals were amplified with three synchronized PORTI-32/MREFA amplifiers 
Table 1. Definition of Individually Adjusted Frequency Bands According to Doppelmayr et al. (1998), Using the Individual Alpha Frequency (IAF) as Anchor Point

\begin{tabular}{lcc}
\hline \hline Frequency band & From & To \\
\hline Theta & $($ IAF $\times 0.4)$ & $($ IAF $\times 0.6)$ \\
Lower alpha1 & $($ IAF $\times 0.6)$ & $($ IAF $\times 0.8)$ \\
Lower alpha2 & (IAF $\times 0.8)$ & $($ IAF $\times 1.0)$ \\
Upper alpha & (IAF $\times 1.0)$ & $($ IAF $\times 1.2)$ \\
Beta & $($ IAF $\times 1.2)$ & $(30 \mathrm{~Hz})$ \\
\hline \hline
\end{tabular}

(Twente Medical Systems BV) and digitized with a sampling rate of $500 \mathrm{~Hz}$.

After the measurement, data were re-referenced to the algebraic mean of the mastoid electrodes (M1 and M2) to obtain a symmetric reference. For artifact reduction, EEG data were rejected off-line whenever the $S D$ of the signal recorded at any electrode exceeded $40 \mu \mathrm{V}$ within a 200 -ms or 800 -ms gliding window. To exclude artifacts caused by eye movements, data were rejected off-line whenever the $S D$ of the EOG signals exceeded $20 \mu \mathrm{V}$ within a 200 -ms sliding window.

Power spectrum analysis was performed using Welch's method of spectral averaging. Artifact-free EEG epochs, lasting $2.048 \mathrm{~s}$ each, were selected and divided into three segments with a length of $1.024 \mathrm{~s}$ and an overlap of $50 \%$. For each condition, single segment power spectra were computed via Fast Fourier Transform (FFT) and then averaged to yield the mean power spectrum. Before FFTcalculation, data segments were windowed using a Hamming function in order to reduce spectral leakage and zero padded to obtain a spectral resolution of $0.448 \mathrm{~Hz}$. Mean band power values were calculated for each condition by averaging power values across frequency bins. On average, 42 epochs per participant entered the grand average of each condition (range: $39-48$ epochs). It has been repeatedly shown that the theta and alpha frequency range (ca. 4-12 Hz) can be subdivided into several functionally distinct narrow frequency bands, the borders of which differ interindividually (e.g., Klimesch, 1999; Klimesch et al., 2005). Therefore, the method of Doppelmayr, Klimesch, Pachinger, and Ripper (1998) was applied to define and adjust five individual frequency bands (theta, lower alpha1, lower alpha2, upper alpha, and beta) using the individual alpha frequency (IAF) as anchor point (see Table 1). The delta band was excluded from analysis to avoid a confusion of the results with slow electrode drifts and small eye movements. The gamma band $(36-40 \mathrm{~Hz})$ was explored without specific hypotheses; however, no effects were found. This is presumably due to the fact that emotional effects within the gamma range are short lasting (e.g., Keil et al., 2001; Muller, Keil, Gruber, \& Elbert, 1999) and cannot be captured with our method, which averages across a $44-\mathrm{s}$ time window.

The IAF of each participant was obtained from the individual power spectra in the baseline condition by averaging the peak frequency measured in the range between 8 and $13 \mathrm{~Hz}$ at electrodes CPZ, PZ, and POZ. Mean IAF of the sample was 10.43 $\mathrm{Hz}(S D$ : $0.85 \mathrm{~Hz}$; IAF range: $9.11-12.29 \mathrm{~Hz}$ ).

To reduce the interindividual variance of absolute power values, individual band power values were normalized using the individual baseline spectra (see Formula). For each participant, each condition $(C)$, electrode $(e)$, and frequency band $(f)$, the individual band power $P_{C}(e, f)$ was divided by the average band power $P_{B}(e, f)$ across $N=61$ scalp electrodes (i.e., except M1 and M2) measured in the baseline condition $(B)$ for the same subject and in the same frequency band $(f)$ :

$$
\hat{P}_{C}(e, f)=\frac{P_{C}(e, f)}{1 / N * \sum_{e=1}^{N} P_{B}(e, f)} .
$$

For statistical analysis the maximum common duration of all musical pieces (i.e., $44 \mathrm{~s}$ ) was evaluated. Additionally, each musical piece was separated into a first (0-22 s) and a second half (22-44 s) to investigate temporal dynamics of brain activity. For the theta band, a two-way repeated measures ANOVA with factors Condition (consonant vs. dissonant) and Time (first vs. second half) was calculated for a fronto-central region of interest (ROI) comprising electrodes AFZ, FZ, and FCZ. For lower alpha1, lower alpha2, upper alpha, and beta, three-way repeated measures ANOVAs with factors Condition, Time, and Hemisphere (left vs. right) were calculated for frontal, centro-parietal, and parieto-occipital ROIs in both hemispheres (frontal: AF4, F4, F8, FC4 vs. AF3, F3, F7, FC3; centro-parietal: C3, C5, CP3, $\mathrm{CP} 5$ vs. C4, C6, CP4, CP6; parieto-occipital: P3, P5, PO3, PO7 vs. $\mathrm{P} 4, \mathrm{P} 6, \mathrm{PO} 4, \mathrm{PO} 8)$.

\section{ECG Recordings and Data Analysis}

The electrocardiogram (ECG) was measured with one electrode placed on each arm and leg as well as six electrodes located on the thorax according to international standards (Lindner, 1999). Instantaneous heart rate (HR) was calculated based on the length of R-R intervals and averaged across participants separately for consonant and dissonant pieces. A prestimulus interval of 1-s length served as baseline as described in the literature (Bradley \& Lang, 2000a). To evaluate initial HR changes as done by Bradley and Lang, a two-tailed $t$ test for paired samples was calculated for the average HR change during the first $8 \mathrm{~s}$ of stimulus presentation. To further investigate the temporal dynamics of HR during the entire musical piece, a two-way repeated measures ANOVA with factors Condition (consonant vs. dissonant) and Time (first vs. second half, each $22 \mathrm{~s}$ ) was calculated.

\section{Tapping Recordings and Data Analysis}

Participants tapped on a $16 \times 8 \times 2 \mathrm{~cm}$ box. Within this box a microphone converted the tapping sound into a voltage. The envelope of this signal was recorded simultaneously with the EEG using an auxiliary input channel of one of the EEG amplifiers. Peaks in this channel were identified as taps. No keyboard or button press was chosen to create a natural tapping situation. To evaluate tapping performance, all intertap time intervals were calculated for each participant and each excerpt. Subsequently, the variance of these intervals was calculated separately and averaged across subjects for each stimulus. To determine whether the participants' tapping accuracy was comparable for both consonant and dissonant pieces as well as for the first and the second half of the excerpts, an ANOVA with factors Condition (consonant vs. dissonant) and Time (first vs. second) was calculated on the tapping variance for all stimulus pairs at the group level.

\section{Results}

Behavioral Ratings

Consonant musical pieces were rated as pleasant, $M=4.74$, $S D=0.51, t(17)=10.21, p<.001$, whereas dissonant excerpts 


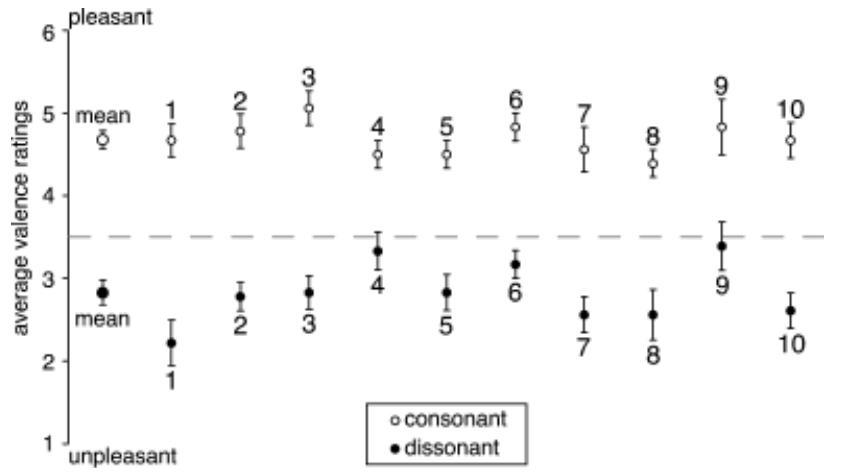

Figure 3. Average valence (average of all consonant and all dissonant pieces [total left] and average for each of the 10 stimulus pairs [1-10]; error bars indicate $S E M$ ). Consonant musical pieces induced pleasant emotions, whereas dissonant pieces induced unpleasant emotions. Consonant pieces were associated with more extreme valence ratings than dissonant pieces.

were rated as unpleasant, $M=2.89, S D=0.73, t(17)=3.50$, $p<.004$ (see Figure 3) as revealed by one-sample $t$ tests (test value $=3.5$, considered as neutral). Accordingly, a two-tailed $t$ test for paired samples on the mean ratings revealed a significant difference between the ratings following consonant or dissonant musical pieces, $t(17)=7.70, p<.001$, replicating previous results also obtained with these stimuli (Koelsch et al., 2006).

Considering 3.5 as the middle of the scale, emotions induced by consonant pieces were rated more extremely ( $M=1.24$ steps higher than $3.5, S D=0.51$ ) than emotions evoked by dissonant pieces $(M=0.61$ steps lower than $3.5, S D=0.73)$. This effect was significant as revealed by a two-tailed $t$ test for paired samples on the absolute differences between mean ratings and 3.5, $t(17)=3.54, p<.004$.

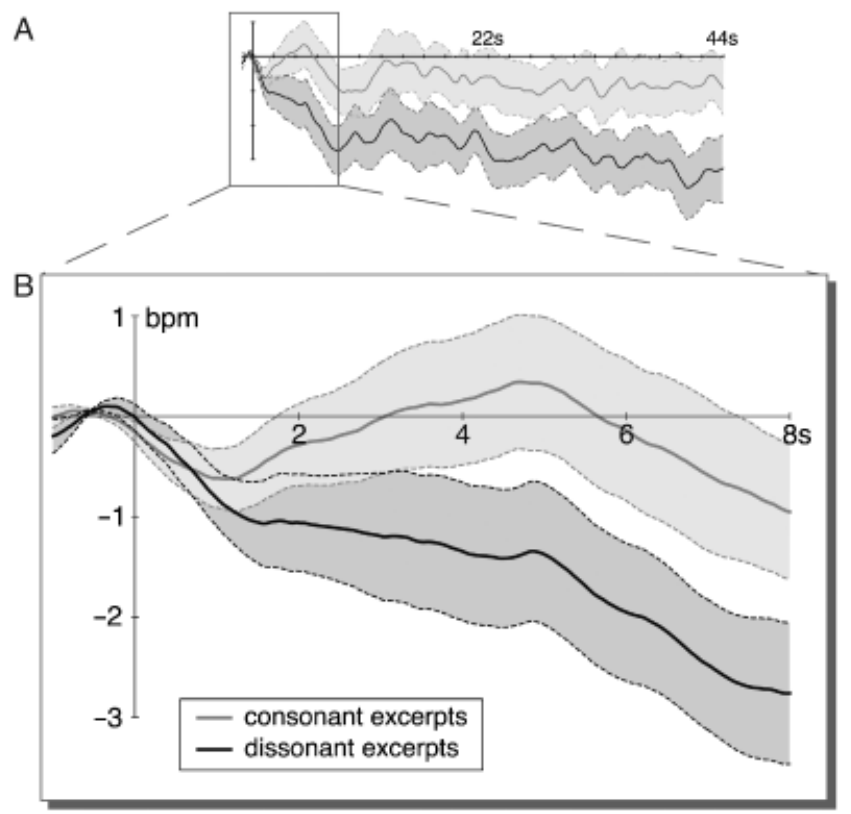

Figure 4. A: Mean heart rate during the course of the musical pieces. HR decreased significantly more strongly during dissonant (unpleasant) compared to consonant (pleasant) pieces, especially during the second half of dissonant excerpts. B: The initial triphasic pattern of heart rate response differed significantly between consonant (pleasant) and dissonant (unpleasant) pieces (solid line: mean; dashed line: SEM).

\section{Tapping}

Participants' tapping performance was comparable between consonant and dissonant pieces as well as between the first and the second half of the excerpts. An ANOVA with factors Condition (consonant vs. dissonant) and Time (first vs. second $22 \mathrm{~s}$ ) on the tapping variance revealed neither main effects (Condition: $p>.2$; Time: $p>.3)$ nor interactions $(p>.2)$. This indicates that participants not only paid attention to the consonant, but also to the dissonant stimuli, and not only to the first but also to the second half of the pieces.

\section{Heart Rate Data}

During the first $8 \mathrm{~s}$ after stimulus onset, the HR showed a triphasic waveform (Figure 4B), resembling the HR changes formerly described by Bradley and Lang (2000a) for emotional picture and sound processing, as well as by Ellis and Simons (2005) for emotional film clips with or without music. That is, stimuli of both categories (consonant and dissonant) evoked a small initial deceleration of HR within the first second, which is taken to reflect an orienting reaction of the listener toward the stimulus. This was followed by a HR acceleration that was stronger for consonant than for dissonant pieces and a secondary deceleration of HR. One-sample $t$ tests with test value $=0$ indicated that the overall HR deceleration within this initial time window $(0-8 \mathrm{~s})$ differed significantly from zero only during listening to dissonant $(-1.48 \mathrm{bpm} ; t[17]=2.93, p<.01)$, but not during consonant pieces $(-0.19 \mathrm{bpm} ; t[17]=0.39, p>.7)$. Furthermore, HR deceleration was significantly greater during the dissonant than during the consonant music, $t(17)=3.27, p<.005$.

To investigate the later dynamics of this HR deceleration in the course of the emotion-inducing music, further analysis included not only the initial $8 \mathrm{~s}$, but a 44-s time period after stimulus onset of each musical excerpt (i.e., the maximum common duration of all musical pieces; see Figure 4A). An ANOVA with factors Condition (consonant vs. dissonant) and Time (first vs. second half, each $22 \mathrm{~s}$ ) revealed that HR during dissonant pieces remained permanently lower than during consonant pieces (main effect of Condition, $F[1,17]=14.85, p<.002)$. Moreover, HR decreased even more strongly during the second (compared to the first) half of dissonant pieces, whereas HR remained stable in the course of consonant excerpts (interaction of Time $\times$ Condition, $F[1,17]=5.19, p<.04$; subsequent two-tailed $t$ tests for paired samples: dissonant pieces [first vs. second half]: $t[17]=2.59$, $p<.02$; consonant excerpts [first vs. second half]: $p>.6$ ). To further investigate the relation between valence and HR deceleration, a correlation between ratings and HR change in a 0-44-s time window was calculated at the stimulus level. This analysis revealed a significant correlation showing that the HR deceleration increased linearly with increasing unpleasantness $(r=.46$, $p<.05, R^{2}=.21$, indicating a medium effect size).

\section{EEG Data: Theta Band, First Level Analysis}

Figure $5 \mathrm{~A}$ depicts maps of the grand-averaged normalized theta power changes with respect to the baseline condition during the first and second half (each $22 \mathrm{~s}$ ) of consonant and dissonant pieces. Blue indicates a power decrease, red a power increase during music compared to baseline (see also Table 2 for mean values and $S E M$ of the theta power with respect to baseline for the mid-frontal ROI).

Theta power over mid-frontal electrodes was greater during consonant compared to dissonant excerpts as reflected by a (marginally) significant main effect of Condition in an ANOVA 


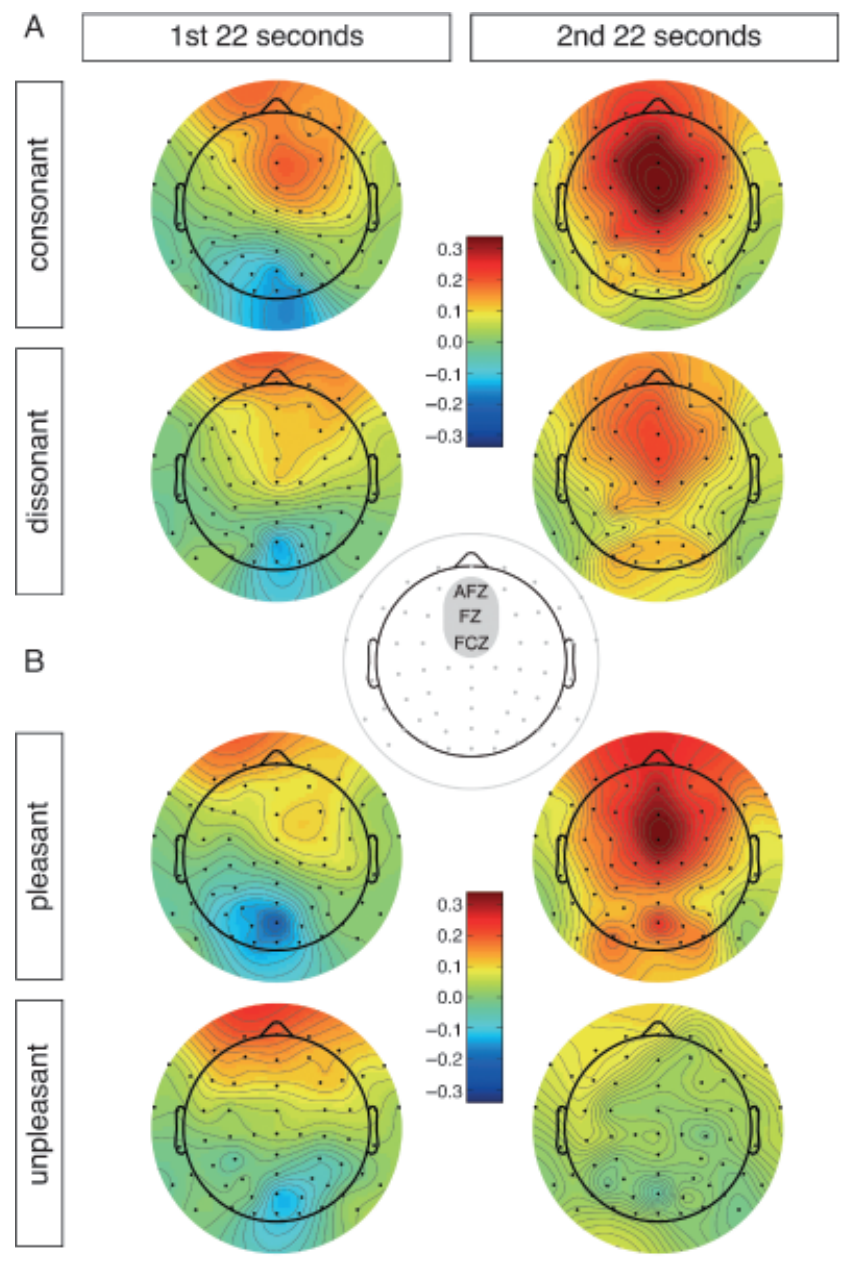

Figure 5. Maps of the average changes of normalized theta power (relative units) with respect to baseline during the first and second halves of consonant and dissonant pieces (A) and during the first and second halves of individually rated (very) (un)pleasant music (B). The shadow in the legend indicates the ROI of the statistical analysis.

with factors Condition (consonant vs. dissonant) and Time (first vs. second) calculated for the mid-frontal ROI (see Table 3 for $F$ and $p$ values as well as for effect size measures partial eta squared $\eta_{p}^{2}$ and omega squared $\omega^{2}$ ). Furthermore, theta power increased significantly toward the end of the musical pieces of both categories as statistically confirmed by a significant main effect of Time. Visually, theta power differed especially during the second half of the excerpts between consonant and dissonant pieces. However, this observation did not reach statistical significance (no interaction).

To further investigate the relation between valence and theta power, a Pearson correlation between ratings and theta power in a 0-44-s time window was calculated at the stimulus level, showing that the mid-frontal theta power increased linearly with increasing pleasantness ratings $(r=.34, p<.08$ [one-tailed], $R^{2}=.12$, indicating a medium effect size).

\section{EEG Data: Theta Band, Second Level Analysis}

According to the participants' ratings, some pieces did not induce strong pleasant or unpleasant emotions but were rather perceived as neutral. This possibly blurred the above found difference be-
Table 2. Summary of Grand-Averaged Changes of Normalized Theta Power with Respect to Baseline ${ }^{\mathrm{a}}$

\begin{tabular}{|c|c|c|c|c|}
\hline & \multicolumn{2}{|c|}{ Consonant } & \multicolumn{2}{|c|}{ Dissonant } \\
\hline & First half & Second half & First half & Second half \\
\hline \multicolumn{5}{|c|}{ First level analysis $(N=18)$} \\
\hline$M$ & 0.176 & 0.349 & 0.110 & 0.219 \\
\hline SEM & 0.077 & 0.083 & 0.065 & 0.088 \\
\hline$t(17)$ & 2.266 & 4.177 & 1.694 & 2.496 \\
\hline$p$ & $.037^{*}$ & $.001^{* * * * *}$ & 0.109 & $.023^{*}$ \\
\hline \multicolumn{5}{|c|}{ Second level analysis $(N=15)$} \\
\hline$M$ & 0.081 & 0.311 & 0.096 & 0.028 \\
\hline SEM & 0.079 & 0.081 & 0.086 & 0.086 \\
\hline$t(14)$ & 1.033 & 3.824 & 1.118 & 0.326 \\
\hline$p$ & .319 & $.002 * *$ & .283 & .749 \\
\hline
\end{tabular}

${ }^{a}$ Asterisks indicate the significance level at which the theta power during music differed from the theta power during baseline as revealed by $t$ tests for paired samples $\left({ }^{*} p<.05,{ }^{* *} p<.01,{ }^{* * * * *} p<.001\right)$.

tween consonant and dissonant pieces. Therefore, in a second level analysis, only tunes with ratings 1 and 2 (i.e., very unpleasant and unpleasant) and 5 and 6 (i.e., pleasant and very pleasant) were analyzed. On average, 30 epochs per participant entered the grand average of each condition (range: 19-30). Three participants had to be excluded from the analysis because they did not rate any of the musical pieces unpleasant or very unpleasant. The results of the 15 remaining participants are shown in Figure 5B and Table 2. In this second level analysis, theta power increased only during the second half of pleasant pieces compared to baseline and remained unchanged in the course of unpleasant excerpts. This was statistically supported by a significant two-way interaction (see Table 3) in an ANOVA with factors Condition (pleasant vs. unpleasant) and Time (first vs. second half), as well as by subsequently calculated $t$ tests for paired samples comparing each half of each music condition with baseline (see Table 2). Furthermore, again a (marginally) significant main effect of Condition was observed.

\section{EEG Data: Lower Alpha1, Lower Alpha2, Upper Alpha, and Beta Band}

First of all, we intended to investigate whether consonant and dissonant music elicited a differentially lateralized decrease of frontal alpha power. Visual inspection of the lower alpha2 and

Table 3. Summary of ANOVAs with Factors Condition and Time for the First and Second Level Analysis of the Theta Effect ${ }^{\mathrm{a}}$

\begin{tabular}{lcccc}
\hline \hline Factor(s) & $F$ values & $p$ values & Partial $\eta_{p}^{2}$ & $\omega^{2}$ \\
\hline First level analysis $(N=18)$ & 4.403 & $.051^{\dagger}$ & .206 & .086 \\
Condition & 5.126 & $.037^{*}$ & .249 & .103 \\
Time & 0.282 & .602 & .016 & - \\
Condition $\times$ Time & $15)$ & & & \\
Second level analysis $(N=144$ & $.061^{\dagger}$ & .229 & .080 \\
Condition & 4.144 & .259 & .090 & - \\
Time & 1.387 & $.046^{*}$ & .256 & .050 \\
Condition $\times$ Time & 4.806 & &
\end{tabular}

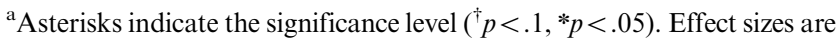
provided at sample level (partial eta squared, $\eta_{p}^{2}$ ), as well as at population level (omega squared, $\omega^{2}$ ). $\omega^{2}$ is only reported for at least marginally significant effects. According to calculations based on Cohen (1992), $\omega^{2}$ greater than .048 and smaller than .26 indicates medium effect sizes. 


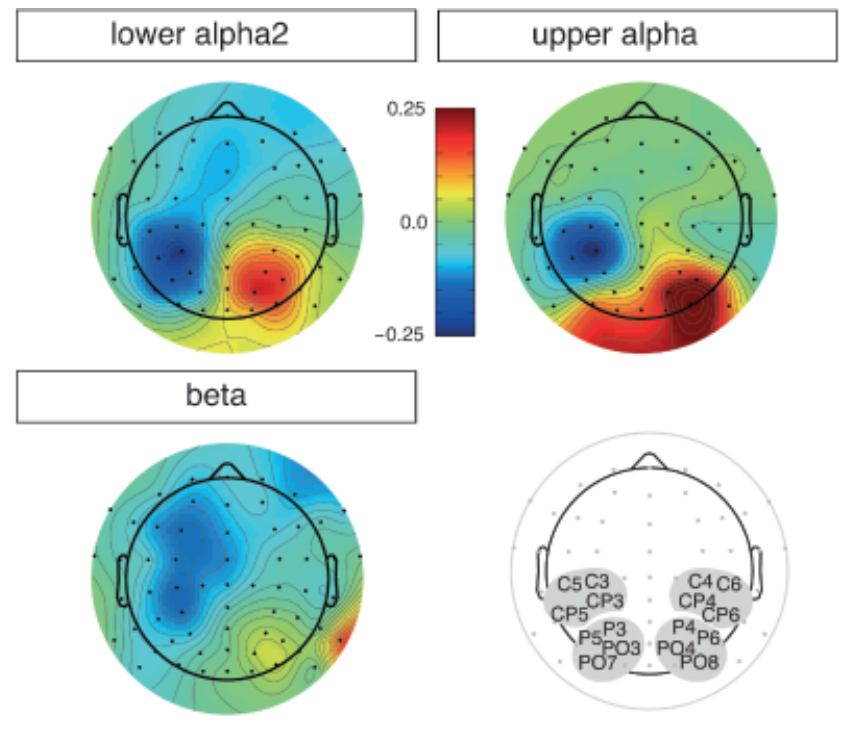

Figure 6. Power changes during listening to music and tapping (i.e., average of the first and second halves of consonant and dissonant musical pieces) compared to baseline in lower alpha2, upper alpha, and beta. No effects of emotional valence or time were found in these frequency bands. However, in association with the tapping task, alpha and beta power decreased over left centro-parietal electrodes (blue) and increased at right parieto-occipital leads (red). The shadows in the legend indicate the ROIs of the statistical analysis.

upper alpha band (corresponding to the frequency range previously investigated) as well as statistical analysis revealed neither power differences between conditions nor a differential lateralization of alpha power during pleasant and unpleasant pieces. No main effects or interactions were found in an ANOVA with factors Condition (consonant vs. dissonant), Time (first vs. second half), and Hemisphere (left vs. right) for the frontal ROIs in any of the two alpha frequency bands. Likewise, no effects were found for lower alphal and beta.

Nevertheless, lower alpha2, upper alpha, and beta (but not lower alpha1) showed a prominent power decrease over left centro-parietal scalp regions and an increase of power over right parieto-occipital leads during listening to music compared to baseline (Figure 6). These effects are most likely due to the motor activity of the right index finger during the tapping task (e.g., Babiloni et al., 1999; Hari \& Salmelin, 1997; Pfurtscheller \& Lopes da Silva, 1999; but see also further below). To statistically analyze this effect, power spectra of both music conditions (first and second half) were combined and compared with the baseline condition by means of ANOVAs with factors Tapping (yes vs. no) and Hemisphere (left vs. right) for the centro-parietal and parieto-occipital ROIs (see Methods). As expected, the observation of the left centro-parietal power decrease during the tapping task was supported by highly significant two-way interactions of Tapping $\times$ Hemisphere in all three frequency bands (lower alpha2: $F[1,17]=12.71, p<.003$; upper alpha: $F[1,17]=11.41, p<.005$; beta: $F[1,17]=27.09, p<.001)$. Similarly, the right parieto-occipital power increase during tapping was supported by (marginally) significant two-way interactions in all three frequency bands (lower alpha2: $F[1,17]=3.12, p<.1$; upper alpha: $F[1,17]=3.88, p<.07$; beta: $F[1,17]=11.52$, $p<.004)$. Furthermore, we investigated whether these effects occurred independently of stimulus category and time course of the musical pieces by means of ANOVAs with factors Condition (consonant vs. dissonant), Time (first vs. second half), and Hemisphere (left vs. right) for the above mentioned ROIs. As expected, because participants tapped during the entire duration of the musical pieces, no significant effects involving Condition or Time (either in centro-parietal, or in parieto-occipital ROIs) were found.

\section{Discussion}

The purpose of the present EEG study was to gain further insights into the brain mechanisms underlying the processing of pleasant and unpleasant musically induced emotions. We investigated the effect of (consonant) joyful musical excerpts and their permanently dissonant counterparts on neuronal oscillatory activity as well as on subjective and psychophysiological indicators of emotional processing.

\section{Behavioral and Heart Rate Data}

The valence ratings indicate that consonant excerpts induced pleasant emotions in listeners, whereas emotions following the dissonant counterparts were perceived as unpleasant, replicating the behavioral findings of a previous study (Koelsch et al., 2006) and of several studies on the relation between sensory dissonance and emotion (Blood et al., 1999; Trainor \& Heinmiller, 1998; Van de Geer, Levelt, \& Plomp, 1962; Zentner \& Kagan, 1996).

The behavioral ratings fit well with the observed HR changes in response to the musical pieces. As predicted, HR decreased significantly more strongly during dissonant than during consonant excerpts. Moreover, the observed pattern of initial HR changes (first $8 \mathrm{~s}$ after stimulus onset) nicely reflects the triphasic pattern of HR response previously described for the processing of emotional pictures (IAPS), sounds (IADS; Bradley \& Lang, 2000a), and film clips (Ellis \& Simons, 2005).

Beyond this initial response, an on-going deceleration of HR was observed in the course of the dissonant but not during the consonant musical excerpts. In previous studies (Bradley \& Lang, 2000a; Lang et al., 1997), HR changes during unpleasant visual and acoustic stimuli were observed to interact with emotional arousal: The overall deceleration of HR during unpleasant stimuli was found to be particularly strong if they were highly arousing compared to moderately arousing. HR changes during pleasant pictures and sounds appeared to be rather independent of arousal. Accordingly, the on-going HR deceleration during dissonant music is attributed to an interaction between the unpleasant emotions and increased arousal during the second half of dissonant musical pieces.

The combined subjective and psychophysiological findings corroborate the prediction that the stimuli used in the present study are capable of inducing pleasant and unpleasant emotions in listeners. Furthermore, this is the first study replicating the typical HR patterns (Bradley \& Lang, 2000a; Ellis \& Simons, 2005; Lang et al., 1997) by means of music as an emotioneliciting stimulus, underlining the general significance of $\mathrm{HR}$ as an index of emotional processing.

\section{Fm Theta Effect}

In the EEG, an increase of Fm theta power was observed, especially during the second half of pleasant musical excerpts. Unpleasant music did not elicit a comparable theta power increase. This finding indicates that Fm theta plays a more important role in emotional processing than previously believed. 
As pointed out earlier, an association between Fm theta and emotion processing is highly likely considering that the sources of this cortical rhythm are thought to be located within the ACC (see Introduction), which is part of the limbic system.

The Fm theta effect was most prominent during the second half of pleasant musical pieces. During both pleasant and unpleasant tunes, emotional arousal was stronger during the second than during the first half (see Methods). This suggests that the affective modulation of the Fm theta power during the pleasant music is not only dependent on the positive valence, but probably requires in addition a certain level of arousal. In other words, a higher arousal level is most probably necessary for the Fm theta effect to occur (see also Bradley \& Lang, 2000b; Cuthbert, Bradley, \& Lang, 1996; Lang et al., 1997, for similar findings for the startle reflex).

The observed Fm theta effect may partly also be related to attentional processes: An emotionally significant stimulus automatically attracts attention (e.g., Lang et al., 1997), and it is, therefore, conceivable that due to the pleasant emotions induced in the course of the consonant pieces, participants listened more attentively to the pleasant-sounding excerpts. The dissonant tunes presumably rather evoked avoidance behavior (see also Koelsch et al., 2006). It thus appears likely that the Fm theta observed in our study is an effect of both emotional and attentional processes. This view is supported by studies reporting that the neural generators of Fm theta are located in the ACC, which has consistently been related to both emotion and attention (e.g., during Stroop tasks; Bush et al., 2000; Devinsky et al., 1995).

In addition to emotion and attention, our results could also relate to other ACC functions, namely motor control (see, e.g., Devinsky et al., 1995). There is a substantial body of evidence that neurons within the dorsal division of the ACC are strongly involved in motor control via dense interconnections with the premotor and motor cortices, the supplementary motor area (SMA; Devinsky et al., 1995; Koski \& Paus, 2000; Paus, 2001), and the spinal cord (Paus, 2001; Picard \& Strick, 1996, 2001). Accordingly, it is conceivable that the special emotional state, which is achieved in the course of the consonant pieces (but not during dissonant excerpts), leads to an additional drive of the motor system, in the sense that pleasant music makes people move (more than unpleasant tunes). This general effect of music has been previously documented by Janata and Grafton (2003). Consequently, the theta effect could at least partially also reflect either the stronger motivation to move, for example, to hum along or to dance (which was, however, not overtly done by the subjects) or the top-down inhibition of this temptation, that is, increased motor control mediated by the ACC. Note that this interpretation is strongly linked to the emotional state of the listener. The theta power increase cannot be interpreted in terms of premotor or primary motor functions per se, because it was only observed during the second half of pleasant excerpts, despite the fact that participants tapped during both the first and second halves of each condition. However, the relation of the Fm theta effect and its function in premotor control remains speculative and has to be elucidated in further studies.

Interestingly, both the Fm theta power and HR differed more strongly between the two stimulus categories during the second (compared to the first) block of the excerpts. Similar findings have been previously reported for strong autonomic responses (Krumhansl, 1997), as well as for brain activations in an f MRI experiment (Koelsch et al., 2006). These findings support the notion that emotional processing has a temporal dynamics, especially when emotions are induced by stimuli that unfold over time (such as music). This observation appears methodologically highly relevant and could be taken into account in future research further investigating the temporal dynamics of emotion and its underlying neural correlates.

\section{Lower Alpha2, Upper Alpha, and Beta}

The present study did not reveal any effects of stimulus valence within the alpha range. Likewise, no hemispheric lateralization as reported in previous EEG emotion studies was found. It is still not clear why some studies do not find evidence for a frontal EEG asymmetry (Hagemann, 2004; Hagemann et al., 1998; Heller \& Nitschke, 1998; Reid et al., 1998). Mediating methodological variables ranging from the reference montage and recorded data length to the handling of artifacts and the choice of participants are currently under debate, but the arguments are still inconclusive. The present study fulfilled the requirements for the investigation of frontal alpha asymmetry, and the reasons for the lack of lateralized effects remain to be specified.

The left central power decrease within the lower alpha2, upper alpha, and beta band is interpreted as a correlate of motor activity due to the finger tapping. This is indicated by the left central scalp distribution of this effect (typical for right-handed movements) and by its independence of both emotional valence and time. Furthermore, this interpretation is supported by studies describing an event-related desynchronization (ERD; i.e., a power decrease) within the alpha and beta frequency ranges during the preparation and execution of finger movements (e.g., Crone et al., 1998; Hari \& Salmelin, 1997; for a review, see Pfurtscheller \& Lopes da Silva, 1999). Note that it is highly unlikely that this effect is due to the music perception. Even if auditory stimulation is known to occasionally decrease the alpha power within auditory areas, this effect occurs bilaterally, contrary to the observed left lateralized alpha power decrease. Furthermore, findings of previous MEG studies (Hari et al., 1997; Salmelin \& Hari, 1994; Tiihonen et al., 1991) suggest that the dipoles of the auditory alpha rhythm are oriented tangentially to the scalp surface and would produce strongest EEG signals in the fronto-central midline as opposed to the present findings.

The right parietal increase of alpha and beta power corresponds to the event-related synchronization (ERS; i.e., a power increase) often reported in association with the desynchronization of the central alpha rhythm during self-paced movements (see Pfurtscheller \& Lopes da Silva, 1999). This antagonist behavior of ERD and ERS, termed "focal ERD/surround ERS," has been interpreted as an activation of task relevant cortical areas (e.g., hand area of the motor cortex) and the simultaneous deactivation of brain areas not involved in the task (e.g., visual areas; see Neuper \& Pfurtscheller, 2001; Pfurtscheller \& Lopes da Silva, 1999).

\section{Limitation}

The present study included only two stimulus categories - consonant and dissonant - and comprised no neutral condition. This makes it somewhat difficult to decide whether an effect emerges from the unpleasantness of the dissonant or from the pleasantness of the consonant musical excerpts (in the present study, we tried to address this issue with correlation analyses). It is important to note that the present design has the major advantage of precisely controlling for the physical properties of the two stimulus categories (see Methods), and it appears highly challenging to create a neutral stimulus class that is as controlled 
as the consonant and dissonant pieces. In future studies, one might also parametrically vary the sensory dissonance to broaden the spectrum of emotional valence (e.g., Blood et al., 1999).

\section{Conclusion}

The present study shows that the typical pattern of HR response as formerly described for the processing of emotional pictures (IAPS), sounds (IADS), and film clips can also be elicited with music, underlining the general significance of HR as an index of emotional processing.
In the EEG, pleasant emotions were accompanied by an increase of Fm theta power, which is interpreted as an effect of emotional processing closely interlinked with attentional functions. This shows that Fm theta power is modulated by emotion more strongly than previously believed, suggesting an enlargement of the concept of Fm theta exceeding pure cognitive functions (such as focused attention), that is, the consideration of its role in emotion processing. The exact ratio of contribution of emotional, attentional, and presumably motor functions to the Fm theta effect remains to be specified.

\section{REFERENCES}

Aftanas, L. I., \& Golocheikine, S. A. (2001). Human anterior and frontal midline theta and lower alpha reflect emotionally positive state and internalized attention: High-resolution EEG investigation of meditation. Neuroscience Letters, 310, 57-60.

Aftanas, L. I., Koshkarov, V. I., Pokrovskaja, V. L., Lotova, N. V., \& Mordvintsev, Y. N. (1996). Pre- and post-stimulus processes in affective task and event-related desynchronization (ERD): Do they discriminate anxiety coping styles? International Journal of Psychophysiology, 24, 197-212.

Aftanas, L. I., Reva, N. V., Savotina, L. N., \& Makhnev, V. P. (2006). Neurophysiological correlates of induced discrete emotions in humans: An individually oriented analysis. Neuroscience and Behavioral Physiology, 36, 119-130.

Aftanas, L. I., Varlamov, A. A., Pavlov, S. V., Makhnev, V. P., \& Reva, N. V. (2001). Affective picture processing: Event-related synchronization within individually defined human theta band is modulated by valence dimension. Neuroscience Letters, 303, 115-118.

Altenmüller, E., Schürmann, K., Lim, V. K., \& Parlitz, D. (2002). Hits to the left, flops to the right: Different emotions during listening to music are reflected in cortical lateralisation patterns. Neuropsychologia, 40, 2242-2256.

Arnolds, D. E. A. T., Lopes daSilva, F. H., Aitink, J. W., Kamp, A., \& Boeijinga, P. (1980). The spectral properties of hippocampal EEG related to behaviour in man. Electroencephalography \& Clinical Neurophysiology, 50, 324-328.

Asada, H., Fukuda, Y., Tsunoda, S., Yamaguchi, M., \& Tonoike, M. (1999). Frontal midline theta rhythms reflect alternative activation of prefrontal cortex and anterior cingulate cortex in humans. Neuroscience Letters, 274, 29-32.

Babiloni, C., Carducci, F., Cincotti, F., Rossini, P. M., Neuper, C., Pfurtscheller, G., et al. (1999). Human movement-related potentials vs. desynchronization of EEG alpha rhythm: A high-resolution EEG study. Neuroimage, 10, 658-665.

Bașar, E. (1999). Brain Function and Oscillations - Volume II: Integrative Brain Function. Neurophysiology and Cognitive Processes. Berlin: Springer.

Bigand, E., Parncutt, R., \& Lerdahl, F. (1996). Perception of musical tension in short chord sequences: The influence of harmonic function, sensory dissonance, horizontal motion, and musical training. Perception \& Psychophysics, 58, 124-141.

Bland, B. H., \& Oddie, S. D. (1998). Anatomical, electrophysiological and pharmacological studies of ascending brainstem hippocampal synchronizing pathways. Neuroscience \& Biobehavioral Reviews, 22, 259-273.

Blood, A. J., \& Zatorre, R. J. (2001). Intensely pleasurable responses to music correlate with activity in brain regions implicated in reward and emotion. Proceedings of the National Academy of Sciences, USA, 98 , $11818-11823$.

Blood, A. J., Zatorre, R. J., Bermudez, P., \& Evans, A. C. (1999). Emotional responses to pleasant and unpleasant music correlate with activity in paralimbic brain regions. Nature Neuroscience, 2, 382-387.

Bradley, M. M., \& Lang, P. J. (1994). Measuring emotion: The SelfAssessment Manikin and the semantic differential. Journal of Behavior Therapy and Experimental Psychiatry, 25, 49-59.

Bradley, M. M., \& Lang, P. J. (1999). International affective digitized sounds. Technical manual and affective ratings. Gainsville, FL: The Center for Research in Psychophysiology, University of Florida.
Bradley, M. M., \& Lang, P. J. (2000a). Affective reactions to acoustic stimuli. Psychophysiology, 37, 204-215.

Bradley, M. M., \& Lang, P. J. (2000b). Measuring emotion: Behavior, feeling, and physiology. In R. D. Lane \& L. Nadel (Eds.), Cognitive neuroscience of emotion (pp. 242-276). Oxford: Oxford University Press.

Bush, G., Luu, P., \& Posner, M. I. (2000). Cognitive and emotional influences in anterior cingulate cortex. Trends in Cognitive Sciences, 4 , 215-222.

Coan, J. A., \& Allen, J. J. (2004). Frontal EEG asymmetry as a moderator and mediator of emotion. Biological Psychology, 67, 7-49.

Cohen, J. (1992). A power primer. Psychological Bulletin, 112, 155-159.

Cook, E. III, \& Turpin, G. (1997). Differentiating orienting, startle, and defense responses: The role of affect and its implications for psychopathology. In P. J. Lang, R. F. Simons, \& M. T. Balaban (Eds.), Attention and orienting: Sensory and motivational processes (pp. 137-164). Mahwah, NJ: Lawrence Erlbaum Associates.

Critchley, H. D., Mathias, C. J., Josephs, O., O'Doherty, J., Zanini, S., Dewar, B. K., et al. (2003). Human cingulate cortex and autonomic control: Converging neuroimaging and clinical evidence. Brain, 126, 2139-2152.

Crone, N. E., Miglioretti, D. L., Gordon, B., Sieracki, J. M., Wilson, M. T., Uematsu, S., et al. (1998). Functional mapping of human sensorimotor cortex with electrocorticographic spectral analysis. I. Alpha and beta event-related desynchronization. Brain, 121, 2271-2299.

Cuthbert, B. N., Bradley, M. M., \& Lang, P. J. (1996). Probing picture perception: Activation and emotion. Psychophysiology, 33, 103-111.

Dalgleish, T. (2004). The emotional brain. Nature Reviews Neuroscience, $5,583-589$.

Davidson, R. J. (1995). Cerebral asymmetry, emotion, and affective style. In R. J. Davidson \& K. Hugdahl (Eds.), Brain asymmetry (pp. 361-387). Cambridge: MIT Press.

Davidson, R. J., Ekman, P., Saron, C. D., Senulis, J. A., \& Friesen, W. V. (1990). Approach-withdrawal and cerebral asymmetry: Emotional expression and brain physiology. I. Journal of Personality and Social Psychology, 58, 330-341.

Davidson, R. J., \& Irwin, W. (1999). The functional neuroanatomy of emotion and affective style. Trends in Cognitive Sciences, 3, 11-21.

Devinsky, O., Morrell, M. J., \& Vogt, B. A. (1995). Contributions of anterior cingulate cortex to behaviour. Brain, 118, 279-306.

Doppelmayr, M., Klimesch, W., Pachinger, T., \& Ripper, B. (1998). Individual differences in brain dynamics: Important implications for the calculation of event-related band power. Biological Cybernetics, $79,49-57$.

Ellis, R. J., \& Simons, R. F. (2005). The impact of music on subjective and physiological indices of emotion while viewing films. Psychomusicology, 19, 15-40.

Gabriel, M., Sparenborg, S. P., \& Stolar, N. (1986). An executive function of the hippocampus: Pathway selection for thalamic neuronal significance code. In R. L. Isaacson \& K. H. Pribram (Eds.), The hippocampus (pp. 1-39). New York: Plenum.

Gevins, A., Smith, M. E., McEvoy, L., \& Yu, D. (1997). High-resolution EEG mapping of cortical activation related to working memory: Effects of task difficulty, type of processing, and practice. Cerebral Cortex, 7, 374-385.

Hagemann, D. (2004). Individual differences in anterior EEG asymmetry: Methodological problems and solutions. Biological Psychology, 67, 157-182. 
Hagemann, D., Naumann, E., Becker, G., Maier, S., \& Bartussek, D. (1998). Frontal brain asymmetry and affective style: A conceptual replication. Psychophysiology, 35, 372-388.

Hari, R., \& Salmelin, R. (1997). Human cortical oscillations: A neuromagnetic view through the skull. Trends in Neuroscience, 20, 44-49.

Hari, R., Salmelin, R., Makela, J. P., Salenius, S., \& Helle, M. (1997). Magnetoencephalographic cortical rhythms. International Journal of Psychophysiology, 26, 51-62.

Heilman, K. M. (1997). The neurobiology of emotional experience. Journal of Neuropsychiatry and Clinical Neurosciences, 9, 439-448.

Heller, W., \& Nitschke, J. B. (1998). The puzzle of regional brain activity in depression and anxiety: The importance of subtypes and comorbidity. Cognition and Emotion, 12, 421-447.

Inanaga, K. (1998). Frontal midline theta rhythm and mental activity. Psychiatry and Clinical Neurosciences, 52, 555-566.

Janata, P., \& Grafton, S. T. (2003). Swinging in the brain: Shared neural substrates for behaviors related to sequencing and music. Nature Neuroscience, 6, 682-687.

Jensen, O., \& Tesche, C. D. (2002). Frontal theta activity in humans increases with memory load in a working memory task. European Journal of Neuroscience, 15, 1395-1399.

Keil, A., Muller, M. M., Gruber, T., Wienbruch, C., Stolarova, M., \& Elbert, T. (2001). Effects of emotional arousal in the cerebral hemispheres: A study of oscillatory brain activity and event-related potentials. Clinical Neurophysiology, 112, 2057-2068.

Klee, M., \& Rall, W. (1977). Computed potentials of cortically arranged populations of neurons. Journal of Neurophysiology, 40, 647-666.

Klimesch, W. (1999). EEG alpha and theta oscillations reflect cognitive and memory performance: A review and analysis. Brain Research Reviews, 29, 169-195.

Klimesch, W., Schack, B., \& Sauseng, P. (2005). The functional significance of theta and upper alpha oscillations. Experimental Psychology, 52, 99-108.

Koelsch, S., Fritz, T., von Cramon, D. Y., Muller, K., \& Friederici, A. D. (2006). Investigating emotion with music: An fMRI study. Human Brain Mapping, 27, 239-250.

Koski, L., \& Paus, T. (2000). Functional connectivity of the anterior cingulate cortex within the human frontal lobe: A brain-mapping meta-analysis. Experimental Brain Research, 133, 55-65.

Krumhansl, C. L. (1997). An exploratory study of musical emotions and psychophysiology. Canadian Journal of Experimental Psychology, 51, 336-353.

Kubota, Y., Sato, W., Toichi, M., Murai, T., Okada, T., Hayashi, A., et al. (2001). Frontal midline theta rhythm is correlated with cardiac autonomic activities during the performance of an attention demanding meditation procedure. Cognitive Brain Research, 11, 281-287.

Lang, P. J., Bradley, M. M., \& Cuthbert, B. N. (1997). Motivated attention: Affect, activation, and action. In P. J. Lang, R. F. Simons, \& M. T. Balaban (Eds.), Attention and orienting: Sensory and motivational processes (pp. 97-135). Mahwah, NJ: Lawrence Erlbaum Associates.

Lang, P. J., Bradley, M. M., \& Cuthbert, B. N. (1999). International Affective Picture System (IAPS): Technical manual and affective ratings. Gainsville, FL: The Center for Research in Psychophysiology, University of Florida.

Lindner, U. K. (1999). Schnellinterpretation des EKG-Ein programmierter Kurs. Berlin: Springer Verlag.

Lopes da Silva, F. H. (1992). The rhythmic slow activity (theta) of the limbic cortex: An oscillation in search of a function. In E. Bașar \& T. H. Bullock (Eds.), Induced rhythms in the brain (pp. 269-283). Boston: Birkhäuser.

Luu, P., \& Tucker, D. M. (2001). Regulating action: Alternating activation of midline frontal and motor cortical networks. Clinical Neurophysiology, 112, 1295-1306.

Luu, P., Tucker, D. M., Derryberry, D., Reed, M., \& Poulsen, C. (2003). Electrophysiological responses to errors and feedback in the process of action regulation. Psychological Science, 14, 47-53.

Luu, P., Tucker, D. M., \& Makeig, S. (2004). Frontal midline theta and the error-related negativity: Neurophysiological mechanisms of action regulation. Clinical Neurophysiology, 115, 1821-1835.

McEvoy, L. K., Pellouchoud, E., Smith, M. E., \& Gevins, A. (2001). Neurophysiological signals of working memory in normal aging. Cognitive Brain Research, 11, 363-376.
Meador, K. J., Thompson, J. L., Loring, D. W., Murro, A. M., King, D. W., Gallagher, B. B., et al. (1991). Behavioral state-specific changes in human hippocampal theta activity. Neurology, 41, 869-872.

Mega, M. S., Cummings, J. L., Salloway, S., \& Malloy, P. (1997). The limbic system: An anatomic, phylogenetic, and clinical perspective. Journal of Neuropsychiatry and Clinical Neurosciences, 9, 315-330.

Mizuki, Y., Kajimura, N., Kai, S., Suetsugi, M., Ushijima, I., \& Yamada, M. (1992). Differential responses to mental stress in high and low anxious normal humans assessed by frontal midline theta activity. International Journal of Psychophysiology, 12, $169-178$.

Muller, M. M., Keil, A., Gruber, T., \& Elbert, T. (1999). Processing of affective pictures modulates right-hemispheric gamma band EEG activity. Clinical Neurophysiology, 110, 1913-1920.

Neuper, C., \& Pfurtscheller, G. (2001). Event-related dynamics of cortical rhythms: Frequency-specific features and functional correlates. International Journal of Psychophysiology, 43, 41-58.

Niedermeyer, E. (1999). The normal EEG of the waking adult. In E. Niedermeyer \& F. H. Lopes da Silva (Eds.), Electroencephalography-Basic principles, clinical applications, and related fields, 4th ed. (pp. 149-173). Baltimore: Williams \& Wilkins.

Nishida, M., Hirai, N., Miwakeichi, F., Maehara, T., Kawai, K., Shimizu, H., et al. (2004). Theta oscillation in the human anterior cingulate cortex during all-night sleep: An electrocorticographic study. Neuroscience Research, 50, 331-341.

Oldfield, R. C. (1971). The assessment and analysis of handedness: The Edinburgh inventory. Neuropsychologia, 9, 97-113.

Onton, J., Delorme, A., \& Makeig, S. (2005). Frontal midline EEG dynamics during working memory. Neuroimage, 27, 341-356.

Paus, T. (2001). Primate anterior cingulate cortex: Where motor control, drive and cognition interface. Nature Reviews Neuroscience, 2, 417-424.

Pfurtscheller, G., \& Lopes da Silva, F. H. (1999). Event-related EEG/ MEG synchronization and desynchronization: Basic principles. Clinical Neurophysiology, 110, 1842-1857.

Pfurtscheller, G., Woertz, M., Supp, G., \& Lopes da Silva, F. H. (2003). Early onset of post-movement beta electroencephalogram synchronization in the supplementary motor area during self-paced finger movement in man. Neuroscience Letters, 339, 111-114.

Phan, K. L., Wager, T., Taylor, S. F., \& Liberzon, I. (2002). Functional neuroanatomy of emotion: A meta-analysis of emotion activation studies in PET and fMRI. Neuroimage, 16, 331-348.

Picard, N., \& Strick, P. L. (1996). Motor areas of the medial wall: A review of their location and functional activation. Cerebral Cortex, 6 , 342-353.

Picard, N., \& Strick, P. L. (2001). Imaging the premotor areas. Current Opinion in Neurobiology, 11, 663-672.

Pizzagalli, D. A., Oakes, T. R., \& Davidson, R. J. (2003). Coupling of theta activity and glucose metabolism in the human rostral anterior cingulate cortex: An EEG/PET study of normal and depressed subjects. Psychophysiology, 40, 939-949.

Reid, S. A., Duke, L. M., \& Allen, J. J. (1998). Resting frontal electroencephalographic asymmetry in depression: Inconsistencies suggest the need to identify mediating factors. Psychophysiology, 35, 389-404.

Salmelin, R., \& Hari, R. (1994). Characterization of spontaneous MEG rhythms in healthy adults. Electroencephalography and Clinical Neurophysiology, 91, 237-248.

Sasaki, K., Tsujimoto, T., Nambu, A., Matsuzaki, R., \& Kyuhou, S. (1994). Dynamic activities of the frontal association cortex in calculating and thinking. Neuroscience Research, 19, 229-233.

Schacter, D. L. (1977). EEG theta waves and psychological phenomena: A review and analysis. Biological Psychology, 5, 47-82.

Schmidt, L. A., \& Trainor, L. J. (2001). Frontal brain electrical activity (EEG) distinguishes valence and intensity of musical emotions. Cognition and Emotion, 15, 487-500.

Sebastiani, L., Simoni, A., Gemignani, A., Ghelarducci, B., \& Santarcangelo, E. L. (2003). Autonomic and EEG correlates of emotional imagery in subjects with different hypnotic susceptibility. Brain Research Bulletin, 60, 151-160.

Sobotka, S. S., Davidson, R. J., \& Senulis, J. A. (1992). Anterior brain electrical asymmetries in response to reward and punishment. Electroencephalography and Clinical Neurophysiology, 83, 236-247.

Steriade, M. (1999). Cellular substrates of brain rhythms. In E. Niedermeyer \& F. H. Lopes da Silva (Eds.), Electroencephalogra- 
phy - Basic principles, clinical applications, and related fields, 4th ed. (pp. 28-75). Baltimore: Williams \& Wilkins.

Suetsugi, M., Mizuki, Y., Ushijima, I., Kobayashi, T., Tsuchiya, K., Aoki, T., et al. (2000). Appearance of frontal midline theta activity in patients with generalized anxiety disorder. Neuropsychobiology, 41, $108-112$.

Tiihonen, J., Hari, R., Kajola, M., Karhu, J., Ahlfors, S., \& Tissari, S. (1991). Magnetoencephalographic $10-\mathrm{Hz}$ rhythm from the human auditory cortex. Neuroscience Letters, 129, 303-305.

Trainor, L. J., \& Heinmiller, B. M. (1998). The development of evaluative responses to music: Infants prefer to listen to consonance over dissonance. Infant Behavior \& Development, 21, 77-88.

Tsang, C. D., Trainor, L. J., Santesso, D. L., Tasker, S. L., \& Schmidt, L. A. (2001). Frontal EEG responses as a function of affective musical features. Annals of the New York Academy of Sciences, 930, 439-442.

Uchida, S., Maehara, T., Hirai, N., Kawai, K., \& Shimizu, H. (2003).

Theta oscillation in the anterior cingulate and beta-1 oscillation in the medial temporal cortices: A human case report. Journal of Clinical Neuroscience, 10, 371-374.

Van de Geer, J., Levelt, W., \& Plomp, R. (1962). The connotation of musical consonance. Acta Psychologica, 20, 308-319.

Vinogradova, O. S. (1995). Expression, control, and probable functional significance of the neuronal theta-rhythm. Progess in Neurobiology, $45,523-583$.

Ward, L. M. (2003). Synchronous neural oscillations and cognitive processes. Trends in Cognitive Sciences, 7, 553-559.

Zentner, M. R., \& Kagan, J. (1996). Perception of music by infants. Nature, 383, 29 .

Zentner, M. R., \& Kagan, J. (1998). Infants' perception of consonance and dissonance in music. Infant Behavior \& Development, 21, 483-492.

(Received July 9, 2006; ACCePted December 5, 2006) 